\title{
Coupling of waveguide mode and graphene plasmons
}

\author{
Jiř́ Petráček ${ }^{1, *}$, Jiři Čtyroký², Vladimír Kuzmiak ${ }^{2}$, Pavel Kwiecien ${ }^{3}$, Ivan Richter ${ }^{3}$ \\ ${ }^{1}$ Brno University of Technology, Faculty of Mechanical Engineering, Institute of Physical Engineering, Technická 2896/2, \\ 61669 Brno, Czech Republic \\ ${ }^{2}$ CAS Institute of Photonics and Electronics, Department of Fiber Lasers and Nonlinear Optics, Chaberská 57, 18251 Prague, \\ Czech Republic \\ ${ }^{3}$ Czech Technical University in Prague, Faculty of Nuclear Sciences and Physical Engineering, Department of Physical Electronics, \\ Břehová 7, 11519 Prague 1, Czech Republic
}

\begin{abstract}
Photonic waveguides with graphene layers have been recently studied for their potential as fast and low-power electro-optic modulators with small footprints. We show that in the optical wavelength range of $1.55 \mu \mathrm{m}$, surface plasmons supported by the graphene layer with the chemical potential exceeding $\sim 0.5 \mathrm{eV}$ can couple with the waveguide mode and affect its propagation. This effect might be possibly utilized in technical applications as a very low-power amplitude modulation, temperature sensing, etc.
\end{abstract}

\section{Introduction}

Unusual electrical and optical properties of novel twodimensional materials like graphene have revolutionized many branches of science and technology. Strong dependence of the surface conductivity of graphene on the chemical potential $\mu_{\mathrm{C}}$, controlled by either doping or applied voltage, makes it possible to modify the effective index of modes propagating in an optical waveguide with a graphene sheet overlay. For the optical communication wavelength range of $1550 \mathrm{~nm}$, a graphene layer with the chemical potential $\mu_{\mathrm{C}}$ below about $0.5 \mathrm{eV}$ introduces a very strong optical attenuation, while for $\mu_{\mathrm{C}}>0.5 \mathrm{eV}$ the attenuation is low when the real part of the mode effective index is changed. In principle, a graphene layer can thus be utilized for both amplitude and phase electro-optic modulation [1-7].

Interestingly, the phase modulation can be accompanied with coupling of the waveguide mode with surface plasmon modes supported by the graphene stripe ("graphene modes"), even at the telecommunication optical wavelength band around $1550 \mathrm{~nm}$ [8].

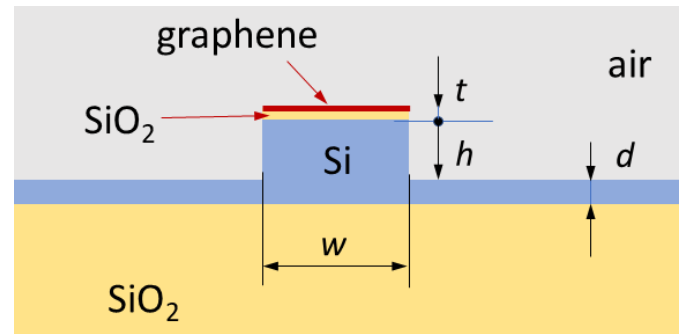

Fig. 1. Si rib waveguide modulator with a graphene layer.

In this paper, we consider a simplified example of a graphene-on-silicon modulator (see Fig. 1), inspired by the design described in [4], and demonstrate the coupling of the fundamental waveguide mode with graphene plasmon modes. We approximately describe the plasmon modes as the superposition two graphene plasmons reflected at the edges of the graphene stripe. Based on our recent comparison of various approaches to numerical modelling of light propagation in a silicon waveguide with graphene overlay [9], we employ a simplified coupled mode theory (CMT) [8] and calculate the complex propagation constant of the quasi-TE mode propagating in the waveguide modulator. We compare the result with a full-wave numerical simulation using the commercial software packet COMSOL Multiphysics. We show that despite the rather crude simplifications used in our implementation of the CMT, the similarity of both results is convincing. Furthermore, the coupling may strongly affect the modulator properties.

\section{Model}

The waveguide structure - the Si rib waveguide modulator with a graphene layer - is shown in Fig. 1. For the calculation we used the following parameters: $h=220 \mathrm{~nm}$, $w=450 \mathrm{~nm}$, and $d=50 \mathrm{~nm}$. Graphene layer is deposited only on the top of the rib waveguide, separated from silicon with a thin $\mathrm{SiO}_{2}$ layer, $t=10 \mathrm{~nm}$. The operating wavelength is $1550 \mathrm{~nm}$.

The optical properties of a graphene monolayer are determined by its complex surface conductivity $\sigma_{s}$, which can be described with an approximate expression presented in $[1,10]$. It is well-known that, in the range of $\mu_{\mathrm{C}}>0.5 \mathrm{eV}$, the positive imaginary part of the surface conductivity strongly prevails (we use the convention $\exp (-i \omega t)$ for time-harmonic quantities) which is the condition allowing propagation of a surface plasmon at

\footnotetext{
* Corresponding author: petracek@fme.vutbr.cz
} 
the interfaces of a graphene layer, considered as an infinitely thin layer with a finite surface conductivity $\sigma_{s}$, sandwiched between two dielectrics.

The surface plasmon propagating along the waveguide axis cannot couple with the guided mode of the silicon waveguide because of a huge (1-2 orders of magnitude) mismatch of their mode effective indices (the effective index of the quasi-TE mode of the bare silicon waveguide calculated with COMSOL is 2.3754). However, the graphene stripe on top of the silicon ridge waveguide, shown in Fig. 1, supports a number of higher order graphene or "nanoribbon" modes [11-14] with smaller propagation constants, and some of them can match with that of the mode of the silicon waveguide. We use an approximate approach for determination of their propagation constants and field distributions. We assumme that similarly to a mode of a planar dielectric waveguide, the graphene modes result from the interference of two plasmons that are propagating under some (complex) angle with respect to the waveguide axis and are reflecting from the stripe edges. The dispersion equation for such modes can be written in the form of the transverse resonance condition (in the $x$ direction in Fig. 2) [8]. Then, reflection of a surface plasmon from the edge of a graphene stripe is assumed to be total, and is modelled with a perfectly magnetic wall. This choice allows for a very simple evaluation of the effective indices and the electromagnetic field distributions of the graphene modes which are quite close to those obtained by using more rigorous COMSOL simulations. It should be noted that some lowest-order modes of the graphene stripe (including central and edge "ribbon plasmons" [14]) are out of scope of this approach. However, these modes cannot couple with the mode of a silicon waveguide due to strong mismatch of their propagation constants.

Coupling of a mode of a silicon waveguide with graphene modes can be considered as mutual coupling among modes of several parallel waveguides. One of the waveguides is the bare silicon waveguide, the other "waveguides" correspond to individual plasmonic modes supported by the graphene stripe. Here, we applied the principles of the complex CMT $[15,16]$ and used it for determination of modes of the composed structure. Our implementation [8] is based on several simplifications; namely, we ignore mutual coupling among various graphene modes and assume symmetrical matrix of coupling coefficients. Such procedure helps significantly simplify the calculation while keeping all aspects important for our analysis.

\section{Results}

Figure 2 shows the distribution of the horizontal electric field intensity component of the fundamental quasi-TE mode of the composed structure, calculated with COMSOL. It will be shown in the following text that the coupling is a resonant effect, therefore for Fig. 2, we used the resonance value of $\mu_{\mathrm{C}}=1.61 \mathrm{eV}$ where the modal loss is maximized, see Fig. 3 (c) (the fundamental quasi-TE mode is labeled as 'Si-Gr' in Fig. 3). The "decoration" of the mode field with the field distribution of the graphene mode is apparent.

Although the dominant electric field component of the plasmons is vertical, for the given structural parameters, there is curiously negligible coupling among the graphene plasmon modes and the TM-polarized waveguide mode. The reason stems from the nature of the coupling mechanism: only electric field components parallel with the graphene layer can contribute to the coupling; apparently, there is no graphene conductivity in the vertical direction.

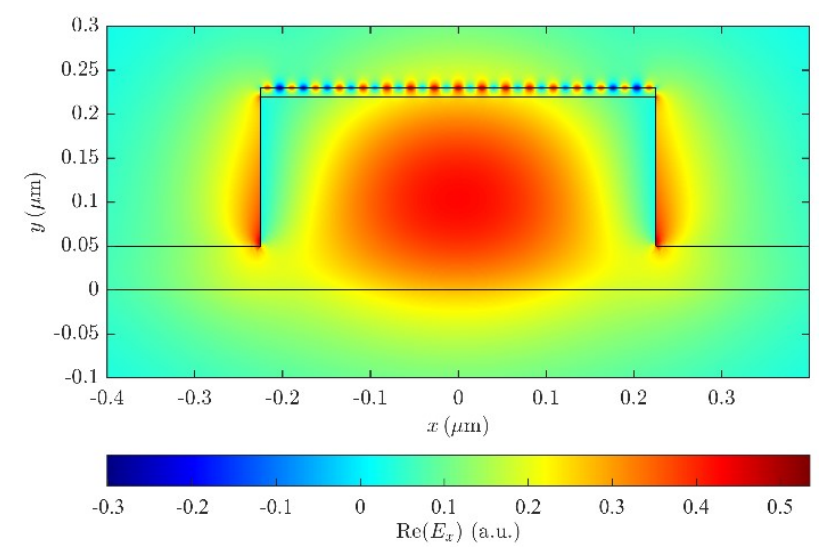

Fig. 2. Distribution of the horizontal $\left(E_{x}\right)$ component of the fundamental quasi-TE mode. The chemical potential of graphene is $\mu_{\mathrm{C}}=1.61 \mathrm{eV}$.

To demonstrate the coupling, we calculated the (complex) mode effective index $N_{\text {eff }}$ for various modes with respect to the change of the chemical potential $\mu_{\mathrm{C}}$ as follows:

(i) Using COMSOL, we first rigorously simulated the bare Si waveguide (with no graphene) and calculated its fundamental quasi-TE mode. Then we considered a graphene layer as a perturbation of the bare waveguide and employed a perturbation technique [9] to determine $N_{\text {eff }}$ for the composed structure. The result is labeled as 'Si' (red line) in Figs. 3 (a), (b), and (c). Note that the perturbation method does not take into account the coupling.

(ii) We used the simplified theory and analytically calculated graphene modes propagating on the graphene stripe on top of the silicon ridge (silica substrate neglected). We considered symmetric graphene modes only (the mirror symmetry of the component $E_{x}$ with respect to the $x=0$ plane; as in Fig. 2), which can couple with the fundamental quasi-TE mode of the bare waveguide. $\operatorname{Re}\left(N_{\text {eff }}\right)$ for the resulting modes are shown in Fig. 3 (a) and marked as 'Gr' (yellow line in Fig. 3 (a) only). In general, for graphene modes, $\operatorname{Re}\left(N_{\text {eff }}\right)$ quickly decreases with increasing $\mu_{\mathrm{C}}$; therefore, only the relevant parts of the dependencies are shown in Fig. 3 (a). The modes exhibit extremely strong modal attenuation that for fixed $\operatorname{Re}\left(N_{\text {eff }}\right)$ decreases with $\mu_{\mathrm{C}}$; for the graphene modes with $\operatorname{Re}\left(N_{\text {eff }}\right)$ displayed in Fig. 3 (a), we observed the decreasing trend with values of $\operatorname{Im}\left(N_{\text {eff }}\right)$ in the range $2<\operatorname{Im}\left(N_{\text {eff }}\right)<5$ (not shown in Fig. 3 ). 
(iii) We used CMT that considers coupling between the waveguide mode (determined in (i)) and graphene modes (determined in (ii)) and calculated the relevant mode of the composed structure; the result is shown in Figs. 3 (b), (c) and marked as 'CMT' (yellow lines in Figs. 3 (b), (c) only).

(a)

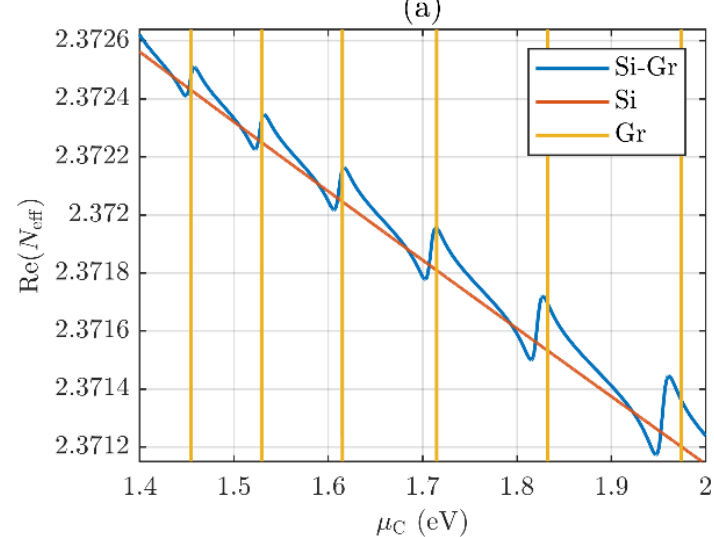

(b)

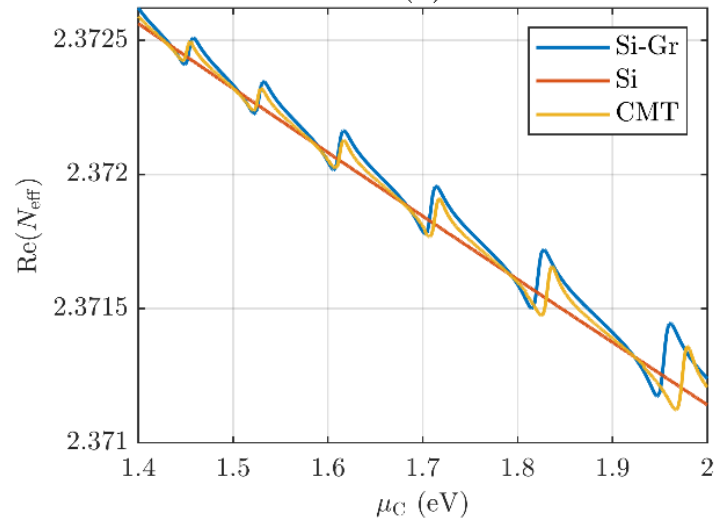

(c)

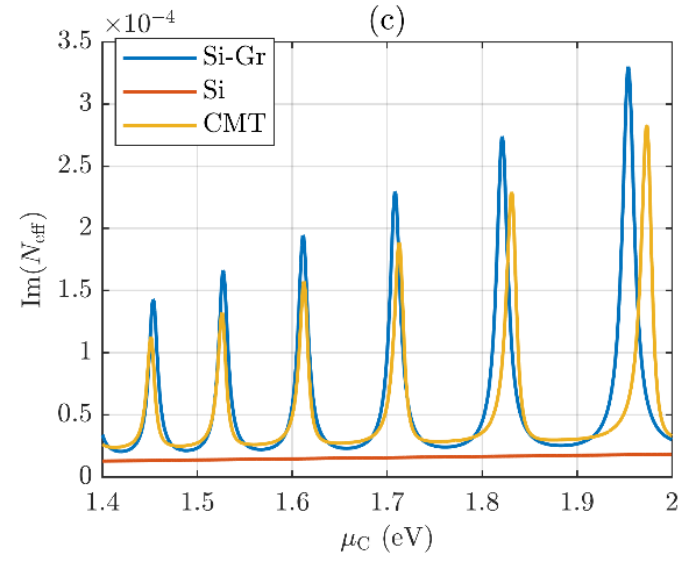

Fig. 3. (a), (b) Real and (c) imaginary parts of the mode effective index $N_{\text {eff }}$ of various modes as functions of the graphene chemical potential $\mu_{\mathrm{c}}$. $N_{\text {eff }}$ for the fundamental quasi-TE mode of the composed structure is marked as follows: 'Si-Gr' rigorous calculation with COMSOL, 'CMT' - CMT calculation, ' $\mathrm{Si}$ ' - the perturbation approach. $N_{\text {eff }}$ for graphene modes is marked as 'Gr'. Only symmetric graphene modes (with the symmetry as the mode in Fig. 2) are shown.

(iv) Finally, we found the modes of the composed structure rigorously with COMSOL; the result for the fundamental quasi-TE mode is shown in Figs. 3 (a), (b), (c) and marked as 'Si-Gr' (blue line). In addition to the fundamental mode, we also display other modes of the composed structure in Fig. 4.

The graphs in Fig. 3 show that the coupling of the silicon waveguide mode with graphene modes has a typical resonant character; see 'Si-Gr' dependencies that reasonably agree with $\mathrm{CMT}$ and thus also provide convincing physical arguments for the presence of the coupling (the perturbation method 'Si' does not take into account the coupling). When the chemical potential is gradually changing, just one of the ' $G r$ ' modes in a time comes into resonance with ' $\mathrm{Si}$ ' mode, i.e., they are phase matched, see Fig. 3 (a). Consequently, at the resonance both the phase and amplitude of the waveguide mode are affected by the coupling. For $\mu_{\mathrm{C}}>1 \mathrm{eV}$ the peaks of $\operatorname{Im}\left(N_{\text {eff }}\right)$ caused by the coupling are of the order of $10^{-4}$, which corresponds to a rather strong modal attenuation of the order of several $\mathrm{dB} / \mathrm{mm}$.

Next, in Fig. 4, we can see a simulated behavior of both real and imaginary parts of the effective indices, with respect to the change of the chemical potential, for the symmetric modes of the composed structure. We display only 'guided' modes with $\operatorname{Re}\left(N_{\text {eff }}\right)$ above the cutoff, $\operatorname{Re}\left(N_{\text {eff }}\right)>N_{\text {eff,c }}=1.6172 ; N_{\text {eff,c }}$ being the effective index of the mode in the lateral waveguide (i.e. the waveguide with the core thickness $d$ ) in Fig. 1. In addition to the fundamental mode (marked as 'Si-Gr'), we observe a rich spectrum of modes that correspond to graphene modes in Fig 3 (a). The 'graphene' modes in Fig. 4 exhibit a strong attenuation that significantly increases when $\operatorname{Re}\left(N_{\text {eff }}\right)$ approaches value of $N_{\text {eff,c }}$ and decreases with increasing $\mu_{\mathrm{C}}$, provided $\operatorname{Re}\left(N_{\text {eff }}\right)$ is kept constant.

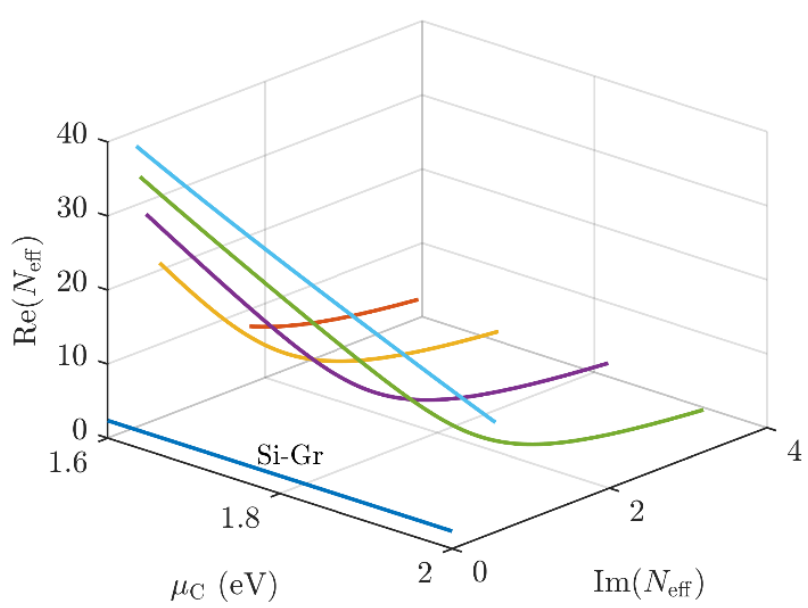

Fig. 4. Modal structure of the Si rib waveguide modulator with a graphene layer with chemical potential $\mu_{\mathrm{C}}$ (rigorous calculation with COMSOL). The curve labeled as 'Si-Gr' describes the fundamental quasi-TE mode (the same mode as ' $\mathrm{Si}-\mathrm{Gr}$ ' dependencies in Fig. 3). Only symmetric modes are shown.

\section{Conclusions}

Properties of graphene plasmons and graphene modes have been previously studied mostly in $\mathrm{THz}$ and infrared frequency ranges. On the other hand, graphene layers on silicon waveguides have been recently very often used in 
the design and construction of photonic devices for modulation, switching, etc. These devices typically operate within the telecommunication band around $1550 \mathrm{~nm}$, where the surface plasmon propagation on graphene layers has attracted much less attention. In this communication we show that in the range of the chemical potential of graphene above $0.5 \mathrm{eV}$, surface plasmons supported by graphene stripes deposited on the top of a silicon waveguides rather strongly affect their guiding properties due to the coupling of graphene plasmon modes with the mode propagating in the waveguide. This effect has been independently studied by both the approximate method based on the CMT, and by "rigorous" numerical simulations using COMSOL. Although the accuracy of the approximate method is not high, it offers a deep insight into the process and contributes to the understanding of the details of the coupling mechanism. The effect has not necessarily been considered harmful for the operation of silicon photonic devices, rather it may be employed in design of specific devices such as low-power modulators and sensors. Although we have focused with our analysis at the near infrared telecom wavelength range, we believe that this effect takes place not only in the near- to mid- IR silicon transparency window, but also in a 'standard' $\mathrm{THz}$ spectral range where the silicon waveguides are being used, too $[6,17,18]$.

This work was financially supported by the Czech Science Foundation under Project 1900062 S and by the Project Center of Advanced Applied Sciences CZ.02.1.01/0.0/0.0/16_019/0000778 - European Structural and Investments Funds - Operational Programme Research, Development, and Education.

\section{References}

1. V. Sorianello, M. Midrio, M. Romagnoli, Design optimization of single and double layer graphene phase modulators in SOI, Optics Express, 23, 6480 (2015)

2. R. Amin, et al., Attojoule-efficient graphene optical modulators, Applied Optics, 57, D130 (2018)

3. G. Kovacevic, C. Phare, S. Y. Set, M. Lipson, S. Yamashita, Ultra-high-speed graphene optical modulator design based on tight field confinement in a slot waveguide, Applied Physics Express, 11, 065102 (2018)

4. V. Sorianello, et al., Graphene-silicon phase modulators with gigahertz bandwidth, Nature Photonics, 120, 40 (2018)
5. V. Sorianello, G. Contestabile, M. Romagnoli, Graphene on silicon modulators, Journal of Lightwave Technology, 38, 2782 (2020)

6. M. Mittendorff, S. S. Li, T. E. Murphy, Graphenebased waveguide-integrated terahertz modulator, ACS Photonics, 4, 316 (2017)

7. Y. S. Tao, H. W. Shu, M. Jin, X. J. Wang, L. J. Zhou, W. W. Zou, Numerical investigation of the linearity of graphene-based silicon waveguide modulator, Optics Express, 27, 9013 (2019)

8. J. Čtyroký, J. Petráček, V. Kuzmiak, P. Kwiecien, I. Richter, Silicon waveguides with graphene: coupling of waveguide mode to surface plasmons, Journal of Optics, 22, 095801 (2020)

9. J. Čtyroký, J. Petráček, P. Kwiecien, I. Richter, V. Kuzmiak, Graphene on an optical waveguide: comparison of simulation approaches, Optical and Quantum Electronics, 52, 149 (2020)

10. F. H. L. Koppens, D. E. Chang, F. J. G. de Abajo, Graphene plasmonics: a platform for strong lightmatter interactions, Nano Letters, 11, 3370 (2011)

11. J. Christensen, A. Manjavacas, S. Thongrattanasiri, F. H. L. Koppens, F. J. G. de Abajo, Graphene plasmon waveguiding and hybridization in individual and paired nanoribbons, ACS Nano, 6, 431 (2012)

12. N. Zhao, Z. X. Zhao, I. A. D. Williamson, S. Boutami, B. Zhao, S. Fan, High reflection from a one-dimensional array of graphene nanoribbons, ACS Photonics, 6, 339 (2019)

13. K. O. Wedel, N. A. Mortensen, K. S. Thygesen, M. Wubs, Edge-dependent reflection and inherited fine structure of higher-order plasmons in graphene nanoribbons Phys. Rev. B, 99, 045411 (2019)

14. H. W. Hou, J. H. Teng, T. Palacios, S. Chua, Edge plasmons and cut-off behavior of graphene nanoribbon waveguides, Optics Communications, 370, 226 (2016)

15. W. P. Huang, J. W. Mu, Complex coupled-mode theory for optical waveguides, Optics Express, 17, 19134 (2009)

16. D. Marcuse, Light Transmission Optics, 2nd ed. (New York: Van Nostrand Reinhold), 1982

17. N. Vermeulen, J. L. Cheng, J. E. Sipe, H. Thienpont, Foundry-compatible SOI waveguides with a graphene top layer for wideband wavelength conversion, SPIE Proceedings 9891, 98911B (2016)

18. A. Locatelli, G. E. Town, C. De Angelis, Graphenebased terahertz waveguide modulators, IEEE Transactions Terahertz Science Technology, 5, 351 (2015) 